\title{
Finely homogeneous computations in free Lie algebras
}

\section{Philippe Andary}

Université de Rouen, Faculté des Sciences, Laboratoire d'Informatique de Rouen, F-76821 Mont-Saint-Aignan Cédex, France

E-Mail: andary@dir.univ-rouen. fr

We first give a fast algorithm to compute the maximal Lyndon word (with respect to lexicographic order) of $L y_{\alpha}(A)$ for every given multidegree $\alpha$ in $\mathbb{N}^{k}$. We then give an algorithm to compute all the words living in $L y_{\alpha}(A)$ for any given $\alpha$ in $\mathbb{N}^{k}$. The best known method for generating Lyndon words is that o: Duval [1], which gives a way to go from every Lyndon word of length $n$ to its successor (with respect to lexicographic order by length), in space and worst case time complexity $O(n)$. Finally, we give a simple algorithm which uses Duval's method (the one above) to compute the next standard bracketing of a Lyndon word for lexicographic order by length. We can find an interesting application of this algorithm in control theory, where one wants to compute within the command Lie algebra of a dynamical system (letters are actually vector fields).

Keywords: Lie algebras, finely homogeneous computations

\section{Introduction}

Let $A=\left\{a_{1}, a_{2}, \ldots, a_{k}\right\}$ be a set with $k$ elements, and $\mathbb{Q}\langle A\rangle$ the associative (non-commutative) algebra on $A$. Defining a Lie bracket $([x, y]=x y-y x)$ on this $\mathbb{Q}$-module turns it into a Lie algebra, and we will denote by $\mathcal{L}(A)$ its Lie subalgebra generated by $A$ (i.e. $\mathcal{L}(A)$ is the free Lie algebra on $A$ and $\mathbb{Q}\langle A\rangle$ its enveloping algebra). A will now be called an alphabet, whose elements are the letters, and $A^{*}$ is the free monoid (the set of all words) over $A$.

We know that $\mathcal{L}(A)$ is a graded $\mathbb{Q}$-module

$$
\mathcal{L}(A)=\bigoplus_{n \geq 1} \mathcal{L}_{n}(A)
$$

where $\mathcal{L}_{n}(A)$ is the submodule of $\mathcal{L}(A)$ generated by homogeneous components with degree $n$. Furthermore, $\mathcal{L}_{n}(A)$ is the direct sum of finely homogeneous submodules $\mathcal{L}_{\alpha}(A)$ with multidegree $\alpha \in \mathbb{N}^{k}$ such that $|\alpha|=n$. Hence we have

$$
\mathcal{L}(A)=\bigoplus_{\alpha \in \mathbb{N}^{k}} \mathcal{L}_{\alpha}(A)
$$


All the classical monomial basis of $\mathcal{L}(A)$ are finely homogeneous (see Reutenauer [2], for example, or Viennot [3]), i.e. their components are finely homogeneous Lie monomials, thus every computation on Lie polynomials (the elements of $\mathcal{L}(A)$ ) is nothing but a computation in some fine homogeneity class.

We want to emphasize the use of a particular basis of $\mathcal{L}(A)$, the Lyndon basis, introduced by Chen $e t$ al. in the late 1950s [4] (the interested reader can find all the details of its construction in Lothaire [5]). For this purpose, we choose a total order $a_{1}<a_{2}<\ldots<a_{k}$ on $A$, which induces a lexicographic order on $A^{*}$. A word $\ell$ is a Lyndon word if it is primitive and minimal in its conjuguacy class. This means that $\ell$ cannot be written as $u^{n}$ for given $u$ in $A^{*}$ and $n \geq 2$, and $\ell \leq v u$ whenever $\ell=u v$. We denote by $L y(A)$ the set of Lyndon words over $A$.

For every Lyndon word $\ell$ we define its (right) standard factorization $\left(\ell^{\prime}, \ell^{\prime \prime}\right)$ as the unique couple of Lyndon words such that $\ell=\ell^{\prime} \ell^{\prime \prime}$ and $\ell^{\prime \prime}$ is of maximal length. Moreover, the recursive Lie bracketing of the standard factorization will be called the (right) standard bracketing of $\ell$, denoted by $[\ell]$.

It would be convenient to define the left standard factorization $(u, v)$ of $\ell$ as the unique couple of Lyndon words such that $\ell=u v$ and $u$ is of maximal length. Then the recursive Lie bracketing of the left standard factorization will be called the left standard bracketing of $\ell$, denoted by $(\ell)$.

Note that $A$ is included in $L y(A)$ and that $[a]=a=(a)$ for every letter $a$. As an exemple, let $A=\{a, b\}$ and $\ell=a a a b a b$, then $\ell$ is Lyndon because it is primitive and $\ell<s$ for each of its proper suffix $s$. The standard bracketings of $\ell$ are

$$
\begin{aligned}
{[\ell] } & =[a,[[a,[a, b]],[a, b]]] \\
(\ell) & =[[a,[a,[a, b]]],[a, b]]
\end{aligned}
$$

It is well known that $L y(A)$ is a factorization of $A^{*}$ and that $[L y(A)]$ is a basis of $\mathcal{L}(A)$ (called the $L y n d o n$ basis of $\mathcal{L}(A))$.

The paper is organized as follows. First, we give a fast algorithm to compute the maximal Lyndon word (with respect to lexicographic order) of $L y_{\alpha}(A)$ for every given multidegree $\alpha$ in $\mathbb{N}^{k}$. We will see that the letters of a Lyndon word which is maximal in its fine homogeneity class are as much 'regularly distributed' as possible.

In the second part, we give an algorithm to compute all the words living in $L y_{\alpha}(A)$ for any given $\alpha$ in $\mathbb{N}^{k}$. The best known method for generating Lyndon words is that of Duval [1], which gives a way to go from every Lyndon word of length $n$ to its successor (with respect to lexicographic order by length), in space and worst case time complexity $O(n)$. It is easy to see that in the special case where $A=\{a, b\}$ and $\ell=a^{k+3} b a^{k-1} b(k>0)$, there are $2^{k-1}-1$ Lyndon words of length $2 k+4$ between $\ell$ and its successor in the same fine homogeneity class (namely $a^{k+2} b a^{k} b$ ). Hence, this method has an exponential worst case time complexity for our purpose.

Finally, we give a simple algorithm which uses Duval's method (the one above) to compute the next standard bracketing of a Lyndon word for lexicographic order by length. We can find an interesting application of this algorithm in control theory, where one wants to compute within the command Lie algebra of a dynamical system (letters actually are vector fields). Standard bracketing are very expansive to compute in this context, and that is why we want to generate them as quickly as possible in the lexicographic order by length. 


\section{Maximal Finely Homogeneous Lyndon Words}

\subsection{Introduction}

Given $\alpha$ in $\left(\mathbb{N}^{*}\right)^{k}$, the question is to find a description of the maximal finely homogeneous Lyndon word with respect to lexicographic order

$$
\mu_{\alpha}(A)=\max \left\{L y_{\alpha}(A)\right\}
$$

We will merely denote it by $\mu_{\alpha}$ when no confusion is possible.

Before answering our question, let us give a short description of the problem's background. Let $\ell$ be a Lyndon word with fine homogeneity $\alpha \in\left(\mathbb{N}^{*}\right)^{k}$; it is well knowr. [5] that

$$
[\ell]=\ell+\sum_{\ell<w} * w
$$

Of course, if $\ell=\mu_{\alpha}$ the only Lyndon word appearing in $[\ell]$ is $\ell$ itself, but the reciprocal may, or may not, be true. Anyhow, this led us to an algorithmic answer to our former question, when $A=\{a, b\}$ in a first time, then for any finite alphabet. Just for fun now, we know that the reciprocal is not true and we were able to find a lot of counter examples (with the help of computers) for which

$$
\operatorname{supp}([\ell]) \cap L y_{\alpha}(A)=\{\ell\}
$$

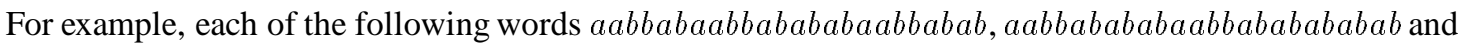
$a a b b a b a b a b a b a b a b a b a b a b a b$ are in $L y_{(12,12)}(\{a, b\})$ and verify equation (5).

\subsection{The Binary Case}

Let us consider the case $k=2$, thus $A=\{a, b\}$. We have the following:

Theorem 1 Given any couple $(n, m)$ of positive integers, the MFHLynd function below returns the maximal Lyndon word in $L y_{(n, m)}(\{a, b\})$ with respect to lexicographic order:

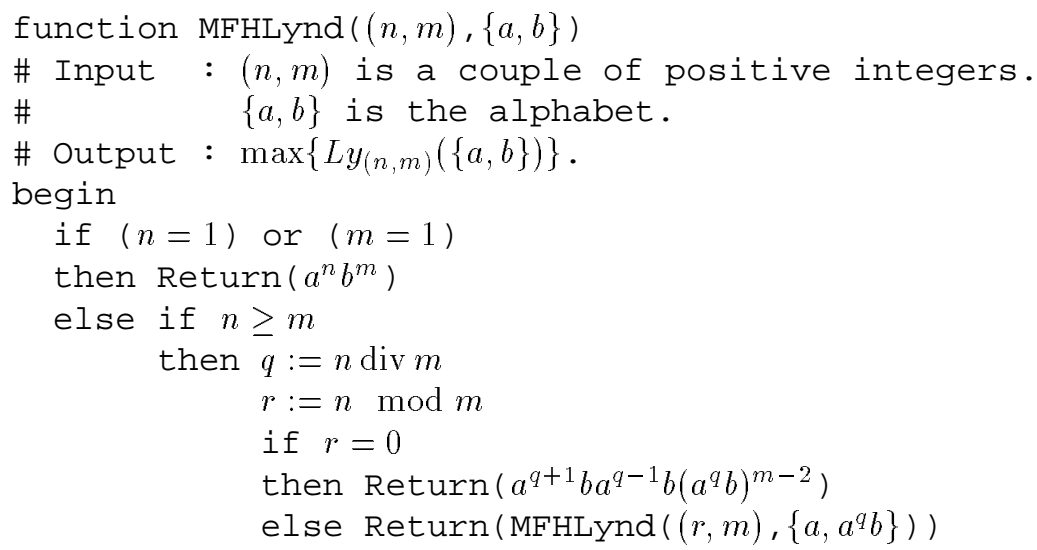




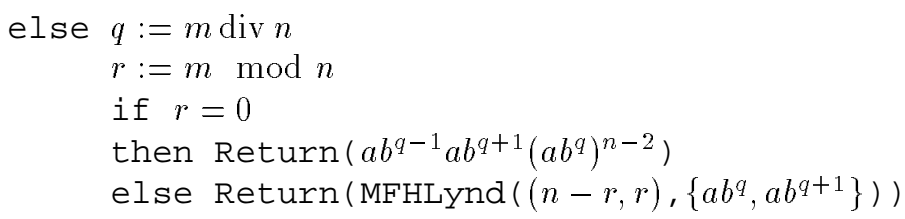

end

Proof. Let $(n, m)$ be any couple of positive integers. We will denote by $\nu$ the result of the MFHLynd function. As this algorithm is recursive, we denote by $n_{i}, m_{i}$ and $A_{i}$ the instances of formal parameters during the $i$-th function call $\left(n_{1}=n, m_{1}=m\right.$ and $\left.A_{1}=\{a, b\}\right)$. Note that the algorithm will terminate in a finite number of steps, say $p$, since through each step $i$ the sum $n_{i}+m_{i}$ will strictly decrease. Furthermore, let us denote by $\mu_{i}$ the word $\mu_{\left(n_{i}, m_{i}\right)}$ at each stage $i$.

If $p=1$ then $n$ is a multiple of $m$, or $m$ is a multiple of $n$, and we obviously have $\nu=\mu_{1}=$ $\mu_{(n, m)}(\{a, b\})$.

So let us consider the case $p>1$, i.e. either $n=q_{1} m+r_{1}$ with $q_{1} \geq 1$ and $0<r_{1}<m$, or $m=q_{1} n+r_{1}$ with $q_{1} \geq 1$ and $0<r_{1}<n$. Then $\left(n_{2}, m_{2}\right)$ is $\left(r_{1}, m\right)$ or $\left(n-r_{1}, r_{1}\right)$, depending on the value of $n$ and $m$, and $A_{2}$ is $\left\{a, a^{q_{1}} b\right\}$ or $\left\{a b^{q_{1}}, a b^{q_{1}+1}\right\}$ accordingly. Of course, $\mu_{2} \leq \mu_{1}$ because $L y\left(A_{2}\right) \subset L y\left(A_{1}\right)$, and if we prove that

$$
\mu_{1} \in A_{2}^{*}
$$

the previous inequality turns to an equality. Now the end of the proof is trivial: we have $\nu=\mu_{p}$, but relation (6) implies

$$
\mu_{1}=\mu_{2}=\ldots=\mu_{p}
$$

Finally, for the proof to be complete, we have to justify relation (6). For this purpose, we will first suppose that $n=q_{1} m+r_{1}$ (thus $A_{2}=\left\{a, a^{q_{1}} b\right\}$ ). We can write

$$
\mu_{1}=a^{q_{1}+\varepsilon_{1}} b \ldots a^{q_{1}+\varepsilon_{m}} b
$$

and one can easily see that $\varepsilon_{1}=1, \varepsilon_{2} \in\{0,1\}$ and $-q_{1} \leq \varepsilon_{i} \leq 1$ for $3 \leq i \leq m$ (since $\mu_{1} \in$ $\left.L y_{(n, m)}(\{a, b\})\right)$. Next, the existence of $h=\min \left\{i: \varepsilon_{i}<0\right\}$ would imply the existence of a Lyndon word $\ell$ in $L y_{(n, m)}(\{a, b\})$ which would be greater than $\mu_{1}$. Indeed, let us write $\mu_{1}=w v_{1} v_{2} \ldots v_{t}$, where $w=a^{q_{1}+1} b \ldots a^{q_{1}+\varepsilon_{h-1}} b a^{q_{1}-x} b\left(x=\left|\varepsilon_{h}\right|\right)$ and the $v_{i}$ 's are defined by the left standard bracketing of $\mu_{1}$

$$
\left(\mu_{1}\right)=\left[\left[\ldots\left[\left[(w),\left(v_{1}\right)\right],\left(v_{2}\right)\right], \ldots\right],\left(v_{t}\right)\right]
$$

Furthermore, we will consider

$$
\begin{aligned}
w^{\prime} & =a^{q_{1}+1} b \ldots a^{q_{1}+\varepsilon_{h-1}-1} b \\
w^{\prime \prime} & =a^{q_{1}-x+1} b
\end{aligned}
$$

Some $v_{i}$ 's are greater than $w^{\prime}$, some are not, but they are all greater than $w$. So we can split those $v_{i}$ between $w$ and $w^{\prime}$ in two words, and apply the same transformation as in $w \rightarrow\left(w^{\prime}, w^{\prime \prime}\right)$. Now construct 
a sequence of Lyndon words, with those $v_{i}$ that are greater than $w^{\prime}$ on the one hand, and the splitted ones $\left(v_{i}^{\prime}, v_{i}^{\prime \prime}\right)$ on the other. Then concatenating this reordered sequence will give us a new Lyndon word which is greater than $\mu_{1}$ (because the former begins with $w^{\prime}$, and the latter with $w$ ). But this fact contradicts the maximality of $\mu_{1}$, so $h$ doesn't exist.

Now, the second case $m>n, m$ not a multiple of $n$, is not substantially different from the previous one. Let us denote by $A^{0}$ the alphabet $A$ equipped with the reverse order $b<a$. Then $\varphi: A \rightarrow A^{0}$ such that $\varphi(a)=b$ and $\varphi(b)=a$ extends to an isomorphism from $A^{*}$ onto $\left(A^{0}\right)^{*}$. Moreover, $\varphi$ is order preserving, and we have clearly

$$
\mu_{(n, m)}(\{a, b\})=\left(\varphi\left(\mu_{(m, n)}(\{a, b\})\right)\right)^{\sim}
$$

where $\left(u_{1} \ldots u_{k}\right)^{\sim}=u_{k} \ldots u_{1}$ is the mirror image of the word $u$.

The analysis of this algorithm is trivial, since its recurrence tree is structurally equivalent to that of Euclide's algorithm for computing the greatest common divisor of $n$ and $m$. So we have

Theorem 2 For given positive integers $(n, m)$, the worst case time complexity for MFHLynd function is $O(\log (\max (n, m)))$.

Proof. See Knuth [6] for instance, Theorem 4.5.3-F and Corollary 4.5.3-L, p. 343.

\subsection{The General Case}

In a second time, we were able to generalize the function MFHLynd to any finite alphabet $A$ in the following manner:

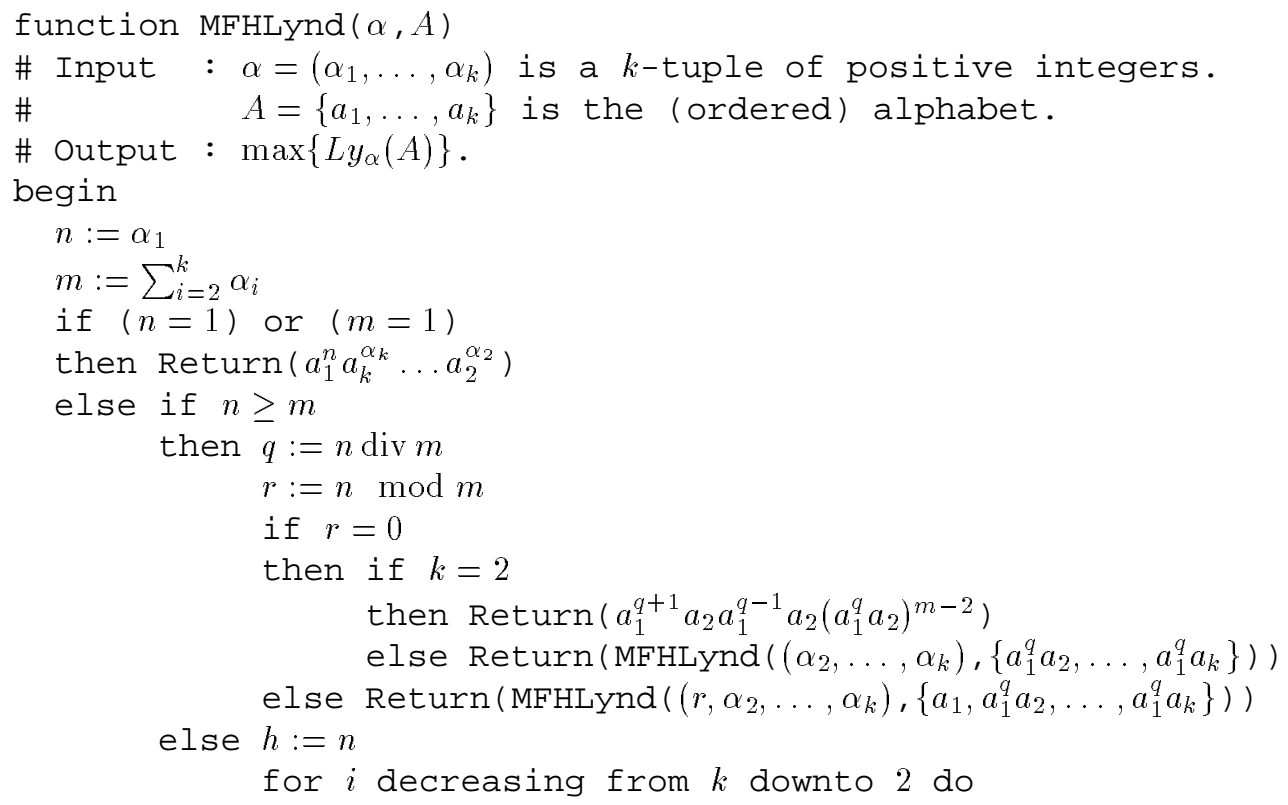




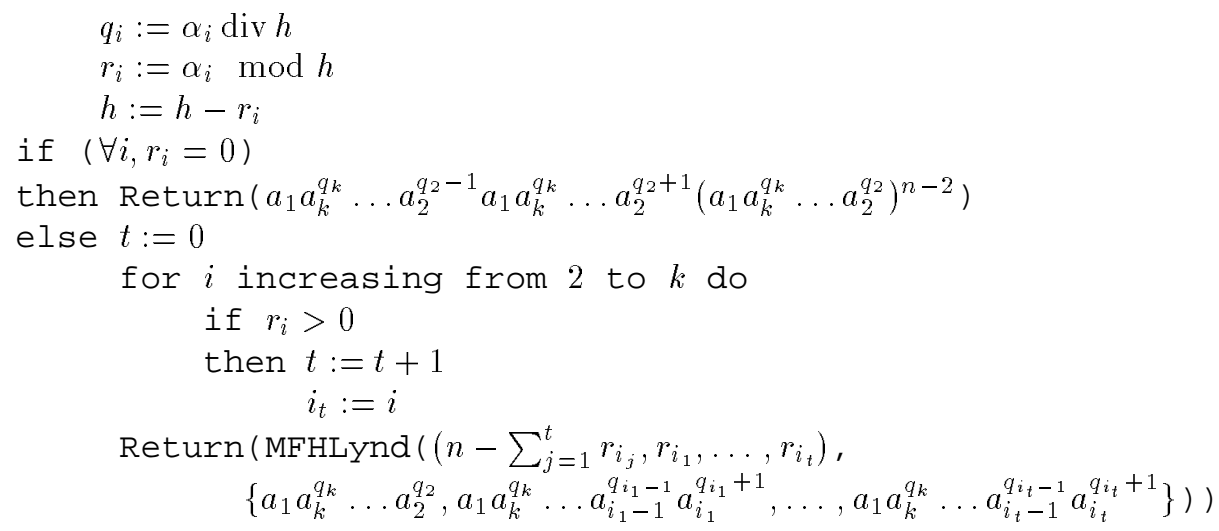

end

We can reasonably expect the complexity of such an algorithm to be a function in the size of the alphabet and in the maximum of the components $\alpha_{i}$, but unfortunately, we were not able to analyse the behaviour of MFHLynd function in this case deeply enough.

\section{Fine Homogeneous Generation of Lyndon Words}

\subsection{Introduction}

Now the problem is to find all words in $L y_{\alpha}(A)$, for every $\alpha$ in $\left(\mathbb{N}^{*}\right)^{k}$. We will need the following well known reducing property (see, for example, Duchamp and Krob [7] for a partially commutative version of this proposition):

Proposition 1 Let $k>1, A=\left\{a_{1}<\ldots<a_{k}\right\}$ and $A^{\prime}=\left(A-a_{k}\right) a_{k}^{*}$. Then $\operatorname{Ly}(A)=\operatorname{Ly}\left(A^{\prime}\right) \cup\left\{a_{k}\right\}$.

Let us see, with an example, how we will dissect Lyndon words to solve our problem. Suppose $\alpha=$ $(3,3)$ and $A=\{a, b\}(k=2)$. By proposition 1 , if $A^{\prime}=a b^{*}$ then $L y(A)=L y\left(A^{\prime}\right) \cup b$. Denoting by $B$ the minimal subset of $A^{\prime}$ such that $L y_{\alpha}(A) \subset B^{*}$, we mean the code

$$
B=\{a, a b, a b b, a b b b\}
$$

We want to find out all Lyndon words on $B$ that are in $L y_{\alpha}(A)$; we know their fine homogeneity, but we don't know how they look like: there can be either two $a$ and one $a b b b$, or one $a$, one $a b$ and one $a b b$. (Note that we cannot take three times $a b$, since Lyndon words are primitive.)

Hence, our initial problem breaks down into two 'smaller' subproblems - find all of the words in $L y_{(2,1)}(\{a, a b b b\})$ and $L y_{(1,1,1)}(\{a, a b, a b b\})$. These subproblems are smaller insofar as the length of the involved Lyndon words are smaller over the new alphabets than over the previous one (here, we have to compare $\{a, a b b b\}$ and $\{a, a b, a b b\}$ with $\{a, b\})$. It is still necessary to detail the way we compute $L y_{(1,1,1)}(\{a, a b, a b b\})$. Here we have $\alpha=(1,1,1), A=\{a, a b, a b b\}(k=3)$ and $B$ is the code

$$
B=\{a, a a b b, a b, a b a b b\}
$$


Every word in $L y_{\alpha}(A)$ is a Lyndon word over $B$, with either one $a a b b$ and one $a b$, or one $a$ and one $a b a b b$. Thus

$$
L y_{(1,1,1)}(\{a, a b, a b b\})=L y_{(1,1)}(\{a a b b, a b\}) \cup L y_{(1,1)}(\{a, a b a b b\})
$$

Clearly, iterating this process will give, in a finite number of steps, every Lyndon word on $\{a, b\}$ with fine homogeneity $(3,3)$. In fact, our algorithm will ultimately construct all alphabets with only one letter which is a word in $L y_{(3,3)}(\{a, b\})$. For example, the subproblems' decomposition tree in this case is

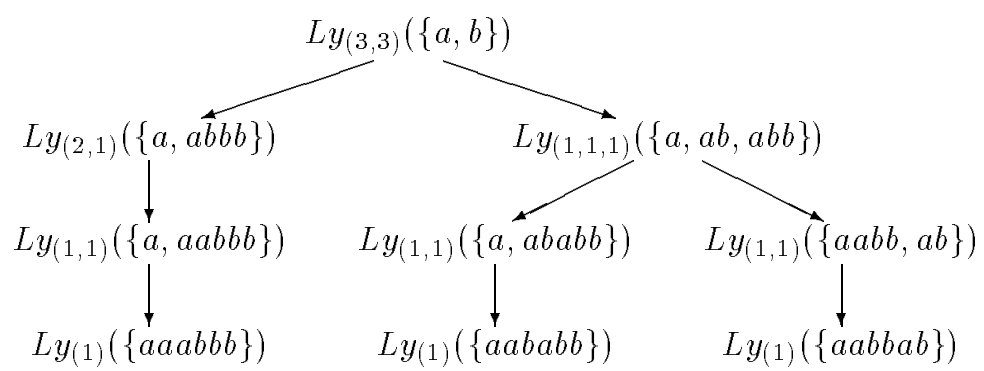

and finally, $L y_{(3,3)}(\{a, b\})=\{a a a b b b, a a b a b b, a a b b a b\}$.

\subsection{Translation in Terms of Partition}

Looking closely at our process gives the feeling that breaking down our problem into smaller ones is exactly the same as finding a set of partitions under some constraints. For example, in the first stage of our previous computation we have searched for the set of elements $x$ in $\mathbb{N}^{4}$ such that $x_{1}+x_{2}+x_{3}+x_{4}=3$, verifying the constraint $x_{1}+2 x_{2}+3 x_{3}+4 x_{4}=6$. We have accepted $(2,0,0,1)$ and $(1,1,1,0)$, refused $(0,3,0,0)$ (since Lyndon words are primitive), and thereby found all the solutions in $x$. Next, during the decomposition of $L y_{(1,1,1)}(\{a, a b, a b b\})$, we have searched for the set of elements $\left(x, x^{\prime}\right)$ in $\left(\mathbb{N}^{2}\right)^{2}$ verifying

$$
\begin{aligned}
x_{1}+x_{2} & =1 \\
x_{1}^{\prime}+x_{2}^{\prime} & =1 \\
x_{1}+2 x_{2}+x_{1}^{\prime}+2 x_{2}^{\prime} & =3
\end{aligned}
$$

Here, again, we have found all the solutions in $\left(x, x^{\prime}\right)$, namely $((1,0),(0,1))$ and $((0,1),(1,0))$.

For a good formulation of the problem in terms of composition and partition, we will need some notations and definitions. For every $x$ in $\mathbb{N}^{p}$, we define

$$
|x|=x_{1}+x_{2}+\ldots+x_{p}
$$

and

$$
\|x\|=x_{1}+2 x_{2}+\ldots+p x_{p}
$$


A composition of the positive integer $n$ into $p$ parts is a $p$-tuple $x$ in $\mathbb{N}^{p}$ such that $|x|=n$. Furthermore, when $\|x\|=n+\lambda$ we say that $x$ is constrained by $\lambda$. We will denote by $\chi(n, p)$ (resp. $\chi_{\lambda}(n, p)$ ) the set of all compositions of $n$ into $p$ parts (resp. the set of all compositions constrained by $\lambda$ ).

A partition of the positive integer $n$ into $p$ parts is a $p$-tuple $y$ in $\left(\mathbb{N}^{*}\right)^{p}$ such that $|y|=n$ and $y_{1} \geq$ $\ldots \geq y_{p} \geq 1$. We will denote by $\pi(n, p)$ the set of all partitions of $n$ into $p$ parts, and by $\pi_{\lambda}(n, p)$ the set of all partitions of $n$ into $p$ parts whose value does not exceed $\lambda$ (thus $\lambda \geq y_{1}$ ).

For consistency with our previous notations, we will call $\bar{x}=\left(x_{i_{1}}, \ldots, x_{i_{t}}\right)$ the composition $x$ without any 0 's (thus, for all $1 \leq j \leq t, x_{i_{j}}>0$ and every other $x_{i}$ are zero), and we will denote by $B_{x}$ the subset of elements of $B$ at the $i_{j}$-th place for lexicographic order $(1 \leq j \leq t)$.

Here is a trivial property which will be helpful for computing the compositions, since we know a constant worst case time complexity algorithm to derive the successor of a partition (see Nijenhuis and Wilf [8]).

Proposition 2 For given $n$, $p$ and $\lambda$, every partition in $\pi_{p}(n+\lambda, n)$ is equivalent to a unique composition in $\chi_{\lambda}(n, p)$.

Proof. The proof is immediate if we see that, given a partition $y$ in $\pi_{p}(n+\lambda, n)$, the corresponding composition $x$ in $\chi_{\lambda}(n, p)$ is such that $x_{i}$ is nothing but the number of parts (in $y$ ) equal to $i$ (for all $i$ in $[1, p])$.

As an example, all the compositions constrained by 3 , of 3 into no more than 4 parts are $(2,0,0,1)$, $(1,1,1,0)$ and $(0,3,0,0)$. Proposition 2 says that they are equivalent to the partitions of 6 into 3 parts not greater than 4 , namely $(4,1,1),(3,2,1)$ and $(2,2,2)$; which will become evident with the notation $1^{2} 4$, 123 and $2^{3}$. For every partition $y$ in this last form, which is equivalent to a composition $x$, we will write $\bar{y}$ the sequence of non zero exponents (for example, if $y=123$ then $\bar{y}=(1,1,1)$ ); so that $\bar{x}=\bar{y}$.

\subsection{The Algorithm}

Now we can state

Theorem 3 Let $k>1, A=\left\{a_{1}<\ldots<a_{k}\right\}$ and $\alpha=\left(\alpha_{1}, \ldots, \alpha_{k}\right) \in\left(\mathbb{N}^{*}\right)^{k}$. Set $B:=\left\{a_{i} a_{k}{ }^{j}: 1 \leq\right.$ $\left.i \leq k-1,0 \leq j \leq \alpha_{k}\right\}, \Phi:=\chi\left(\alpha_{k}, k\right)$ and for each $\phi$ in $\Phi$ :

$$
X_{\phi}:=\chi_{\phi_{1}}\left(\alpha_{1}, \alpha_{k}+1\right) \times \ldots \times \chi_{\phi_{k-1}}\left(\alpha_{k-1}, \alpha_{k}+1\right) .
$$

Then we have

$$
L y_{\alpha}(A)=\bigcup_{\phi \in \Phi} \bigcup_{x \in X_{\phi}} L y_{x}(B)
$$

Proof. On the one hand, we will study the case $k=2$, so we consider $A=\{a, b\}, \alpha=(n, m)$ and $B=\left\{a b^{j}: 0 \leq j \leq m\right\}$. There is $m+1$ elements in $B$, and to each composition $x \in \mathbb{N}^{m+1}$, we associate the fine homogeneous class of words in $B^{*}$

$$
F_{x}=\left\{w \in B^{*}:|w|_{a b^{j}}=x_{j}, 0 \leq j \leq m\right\} .
$$

Since $F_{x}$ is never empty, there is at least one Lyndon word in $F_{x}$ (note that, by Proposition $1, \operatorname{Ly}(B) \subset$ $L y(A))$ and we want to determine those $x$ such that $F_{x}$ contains a Lyndon word in $L y_{\alpha}(A)$ - thus every 
Lyndon word in $F_{x}$ will belong to $L y_{\alpha}(A)$. Of course, $L y_{\alpha}(A)$ will be the union set of $L y_{x}(B)$ over all these $x$. But it is clear that the only criteria for $x \in \mathbb{N}^{m+1}$ to be choosen are $|x|=n$ and $\|x\|=n+m$. So $x$ must be a composition of $n$ into no more than $m+1$ parts, constrained by $m$, and the theorem is proved when $k=2\left(\right.$ with $\Phi=\{(m)\}$ and $\left.X_{(m)}=\chi_{m}(n, m+1)\right)$.

On the other hand, we will now consider the case $k>2$. Then we have $B=\left\{a_{i} a_{k}{ }^{j}: 1 \leq i \leq\right.$ $\left.k-1,0 \leq j \leq \alpha_{k}\right\}$ and $|B|=k\left(\alpha_{k}+1\right)$. Here, the problem is to find all $x=\left(x^{(1)}, \ldots, x^{(k-1)}\right) \overline{\text { in }}$ $\left(\mathbb{N}^{\alpha_{k}+1}\right)^{k-1}$ such that

$$
\left\{\begin{aligned}
\left|x^{(i)}\right| & =\alpha_{i} \quad(1 \leq i \leq k-1) \\
\sum_{i=1}^{k-1}\left\|x^{(i)}\right\| & =|\alpha|
\end{aligned}\right.
$$

Thus we must have, for all $i$, the inequalities

$$
\alpha_{i} \leq\left\|x^{(i)}\right\| \leq \alpha_{i}+\alpha_{k}
$$

and it is clear now that the set of solutions for system (20) is exactly the union set over $\phi \in \chi\left(\alpha_{k}, k\right)$ of the $|B|$-tuple $x$ of (non-negative) integers verifying

$$
\left\{\begin{aligned}
\left|x^{(i)}\right| & =\alpha_{i} \\
\left\|x^{(i)}\right\| & =\alpha_{i}+\phi_{i}
\end{aligned}\right.
$$

for all $1 \leq i \leq k-1$.

From Theorem 3 and Proposition 2, we can deduce the function FHGLynd, which takes as input an alphabet $A$ and a multidegree $\alpha$, and outputs the set of elements in $L y_{\alpha}(A)$

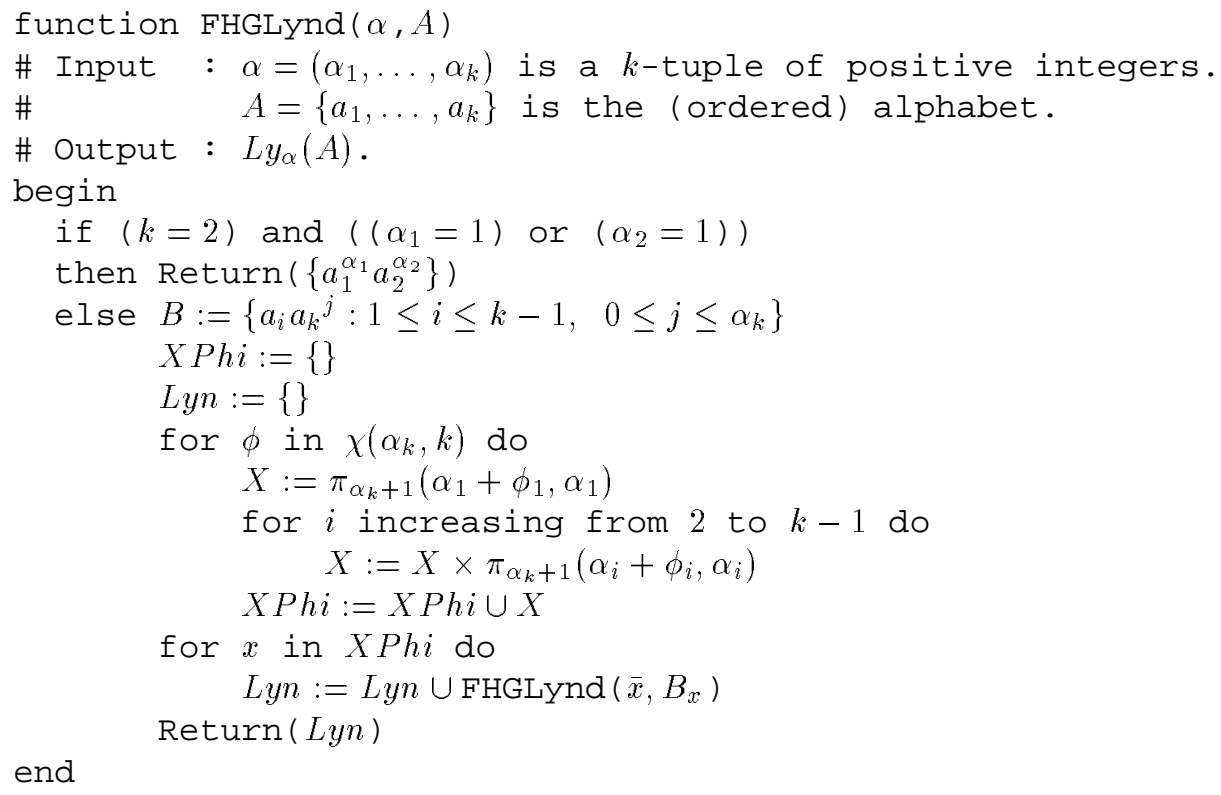




\subsection{Tests on the Execution Time}

Except for the FHGLynd function, the only algorithm at hand for computing the homogeneity class of Lyndon words could be one derived from Duval's method. As we will see in the next section, this method allows us to go from one Lyndon word to the (lexicographically) next one with the same length. Thus, a new function, say FHGL, is born: given an alphabet $A$ with $k$ letters and a homogeneity vector $\alpha$ (with $n=|\alpha|$ ), construct the set $L y_{\alpha}(A)$ by iterative application of Duval's method, picking up those words whose homogeneity is $\alpha$. However, we have to bear in mind that this is an exponential algorithm, since the number of Lyndon words whose length is $n$ is $O\left(\frac{k^{n}}{n}\right)$. We wanted to get a feeling on the complexities of both methods. For this purpose, both algorithms have been implemented in Maple V.3 on an SS10 workstation under Solaris 2.3 operating system.

The first time we found out that, for the generation of a given homogeneity class of Lyndon words, the FHGLynd function is more efficient than FHGL.

The second time, we decided, given a finite alphabet $A$ of size $k$ and an integer $n$, to generate all the words in $L y_{n}(A)$, first in lexicographic order by Duval's method (algorithm D), and then by homogeneity classes with our function (algorithm $A$ ). Although the average running time of A per homogeneity class becomes gradually smaller than the running time of $\mathrm{D}$ while $k$ and/or $n$ grow, algorithm $\mathrm{A}$ does not seem to be linear in $n$, since algorithm $\mathrm{D}$ does, and their execution time ratio increases.

Hence, it is obvious that these two algorithms are devoted to different problems: FHGL is well suited for lexicographic enumeration of Lyndon words of given length, while F HGLynd is just right for homogeneity generation of Lyndon words.

\section{Generating the Lyndon Basis}

\subsection{Introduction}

We want here to generate the standard bracketing of Lyndon words for lexicographic order by length. We know an efficient algorithm, due tc Duval [1]: which gives the next Lyndon word for lexicographic order by length with a constant average time complexity [9]. The idea is quite simple: adapt Duval's method to obtain the index of the standard factorization in addition to the Lyndon word, of course, preserving time complexity; then a convenient tree data structure could be used to recursively store all the standard factorizations.

\subsection{Duval's Theorem}

We will write ' $\leq$ ' and ' $\preceq$ ' for lexicographic order and lexicographic order by length, respectively. These total orders on $A^{*}$ are defined as follows:

$$
\begin{aligned}
& u \leq v \quad \text { iff } \begin{cases} & v \in u A^{*}, \\
\text { or } \quad \begin{array}{rl} 
\\
& \left(\exists r, s, t \in A^{*}\right)(\exists a, b \in A)(u=r a s, v=r b t, a<b)
\end{array}\end{cases}
\end{aligned}
$$

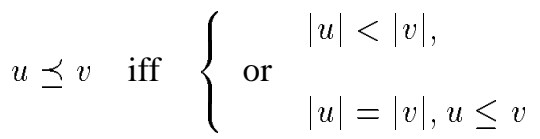

For all Lyndon word $\ell$, we will denote by $S(\ell)$, when it makes sense, its successor, for lexicographic order, which is not in $\ell A^{*}$. It is computed as follows: remove all terminal maximal letters of $\ell$, then 
replace the last non-maximal letter by its successor in $A$. Notice that $S(\ell)$ has always a smaller size than $\ell$. For example, on $A=\{a, b, c\}, S(a a a c c)$ is $a a b$ (not $a a b a b !)$, and $S(a a a b b)$ is $a a a b c$. Duval proves that

Theorem 4 If $\ell$ is a Lyndon word with length $n$, which is not maximal in its fine homogeneous class; if $w$ is the following Lyndon word of $\ell$ for ' $\preceq$ ', then we have:

$$
w= \begin{cases}S(\ell) & \text { if }|S(\ell)|=n \\ u_{1}^{q_{1}} \ldots u_{t}^{q_{t}} & \text { otherwise }\end{cases}
$$

where the $u_{i}$ are Lyndon words derived from prefixes of $\ell$.

More precisely, the $u_{i}$ are defined as follows (where $u[1 . . j]$ is the prefix of $u$, whose length is $j$ ):

$$
\begin{array}{llll}
u_{1}=S(\ell), & d_{1}=\left|u_{1}\right|, & q_{1}=(n-1) \operatorname{div} d_{1}, & r_{1}=1+(n-1) \bmod d_{1}, \\
u_{2}=S\left(u_{1}\left[1 . r_{1}\right]\right), & d_{2}=\left|u_{2}\right|, & q_{2}=r_{1} \operatorname{div} d_{2}, & r_{2}=r_{1} \bmod d_{2}>0, \\
& & & \\
u_{t}=S\left(u_{1}\left[1 . . r_{t-1}\right]\right), & d_{t}=\left|u_{t}\right|, & q_{t}=r_{t-1} \operatorname{div} d_{t}, & r_{t}=r_{t-1} \bmod d_{t}=0 .
\end{array}
$$

Let us have a feeling on the behaviour of the underlying algorithm on the Lyndon word $\ell=a^{3} b a b^{10}$ (over $A=\{a, b\}$ ). The first prefix we compute is $\left(a^{3} b^{2}\right)^{2}$, since $S(\ell)=a^{3} b^{2}$, and because $r_{1}$ must be positive. Then we get $\left(a^{3} b^{2}\right)^{2} a^{2} b$ since $S\left(a^{3} b^{2}\right)=a^{2} b$, and finally, we compute $S\left(a^{2}\right)=a b$ giving $w=\left(a^{3} b^{2}\right)^{2} a^{2} b a b$.

This algorithm is $O(|\ell|)$ in worst case time complexity, and Berstel and Pocchiola [9] have proved it to be optimal in average time complexity (actually, they give the asymptotic bound $(k+1) / k$, where $k$ is the cardinal of $A$ ).

\subsection{Adapted Duval's Algorithm}

We have seen that Duval constructs the next Lyndon word by computing successive prefixes, thus we had the natural idea to use the left standard factorization instead of the right one, as usual. So, if we denote by $i(u)$ the index of the left standard factorization of a Lyndon word $u$, and with the notations of Theorem 4 , we have

Theorem 5 If $w=S(\ell)$, then $i(w)=i(\ell) ;$ otherwise if $w=u_{1}{ }^{q_{1}} u_{2}$, then $i(w)=d_{1}$, else $w=$ $u_{1}^{q_{1}} \ldots u_{t}^{q_{t}}$ and $i(w)=n-d_{t}$.

Proof. First, we have to notice that, for all $1<i \leq t$, the relation $u_{i}=S\left(u_{1}\left[1 \ldots r_{i-1}\right]\right)$ implies the existence of the integer $d_{i}$ in $\llbracket 1, r_{i-1} \rrbracket$ and the letter $\lambda_{i}=\operatorname{succ}\left(u_{i-1}\left[d_{i}\right]\right)$ such that $\left(\operatorname{set} u_{0}=\ell\right)$

$$
u_{i}=u_{i-1}\left[1 . . d_{i}-1\right] \lambda_{i}
$$

So we have

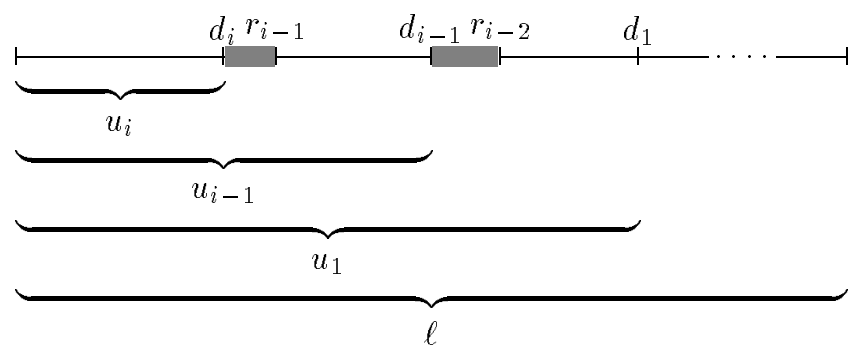


where grayed zones means the suppression of maximal letters.

Now we can carry through the proof. If $w=S(\ell)$ this is because $|S(\ell)|=n$, that is $S(\ell)=$ $\ell_{1} \ldots \ell_{n-1} \operatorname{succ}\left(\ell_{n}\right)$ with $\ell_{n}<z$. In this case $i(w)$ is obviously $i(\ell)$.

Otherwise, there are two cases left. On the one hand, when $w=u_{1}{ }^{q_{1}} u_{2}$ we must have $i(w)=$ $d_{1}$ because of the preliminary remark. On the other hand, in the general case $(t>2$, or $t=2$ and $q_{2}>1$ ), the longest prefix of $w$ which is Lyndon, is at least as long as $u_{1}^{q_{1}} \ldots u_{t-1}{ }^{q_{t-1}} u_{t} q_{t}-1$, since $u_{1}<u_{2}<\ldots<u_{t}$. But according to the preliminary remark, it cannot be longer than this word. Hence $i(w)=\sum_{i=1}^{t-1} q_{i} d_{i}+\left(q_{t}-1\right) d_{t}=n-d_{t}$.

As an example, let us consider $A=\{a, b\}$ and $n=5$. Then we have the following table:

\begin{tabular}{ccc}
\hline \hline$(\ell, i(\ell))$ & $w=u_{1}{ }^{q_{1}} \ldots u_{t}^{q_{t}}$ & $i(w)$ \\
\hline$(a a a a b, 1)$ & $(a a a b)(b)$ & 4 \\
$(a a a b b, 4)$ & $(a a b)(a b)$ & 3 \\
$(a a b a b, 3)$ & $(a a b b)(b)$ & 4 \\
$(a a b b b, 4)$ & $(a b)^{2}(b)$ & 2 \\
$(a b a b b, 2)$ & $(a b b)(b)^{2}$ & 4 \\
\hline \hline
\end{tabular}

for the four first lines $i(w)$ is $d_{1}$, since $t=2$ and $q_{2}=1$, but for the fifth it is $n-d_{2}=4$ since $q_{2}>1$.

Now, for the sake of completeness, let us give the slight improvement of Duval's algorithm

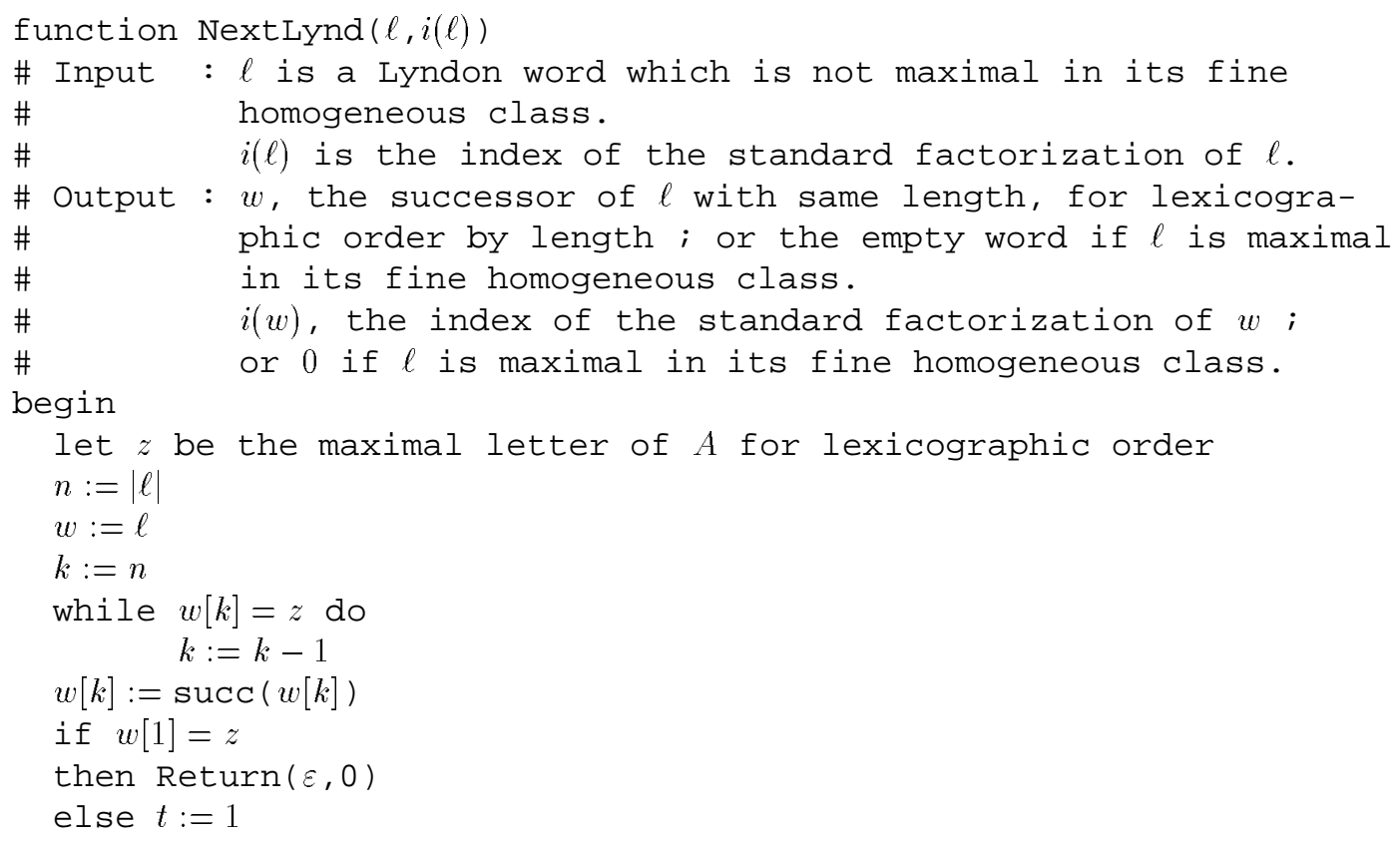




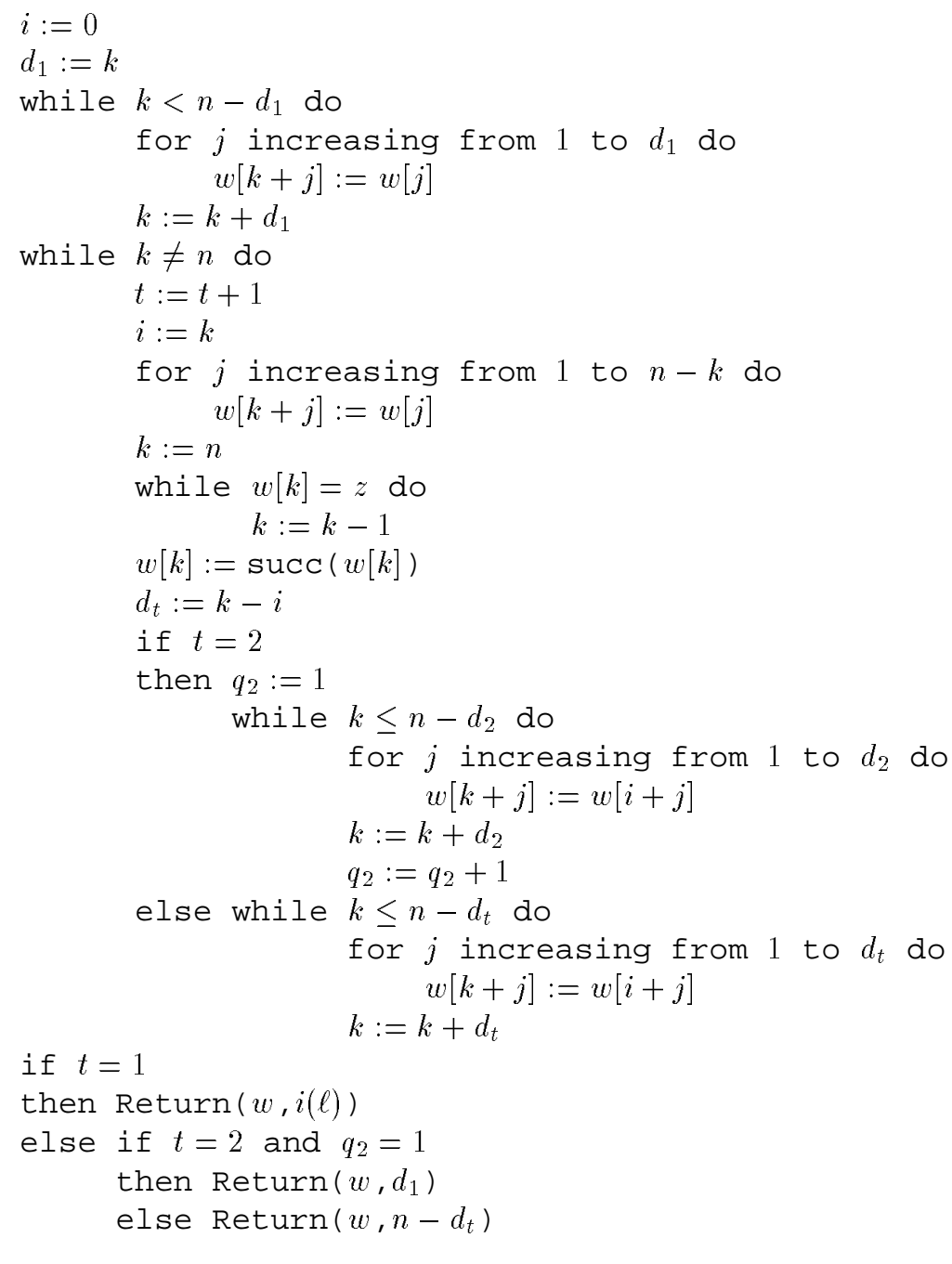

where $u[j]$ means the $j^{\text {th }}$ letter of $u$, and $\operatorname{succ}(\lambda)$ is the following letter of $\lambda$ in $A$.

It is clear that the complexity is the same as for the original algorithm, since we have added only two tests in the function's body (and also the use of four new integer variables).

\section{References}

[1] Duval, J.-P. (1988). Génération d'une section des classes de conjuguaison et arbre des mots de Lyndon de longueur bornée. Theor. Comput. Sci. 60, 255-283. 
[2] Reutenauer, C. (1993). Free Lie Algebras. London Mathematical Society Monographs, new series, Vol. 7. Academic Press.

[3] Viennot, X. G. (1978). Algèbres de Lie libres et monoïdes libres. Lecture Notes in Mathematics 691. Springer-Verlag.

[4] Chen, K. T., Fox, R. H. and Lyndon, R. C. (1958). Free differential calculus, IV. The quotient groups of the lower central series. Ann. Mathematics 68, 81-95.

[5] Lothaire, M. (1983). Combinatorics on words. Encyclopedia of Mathematics 17. Addison-Wesley.

[6] Knuth, D. E. (1981). The Art of Computer Programming, vol. 2: Semi-numerical algorithms (2nd ed.) Addison-Wesley.

[7] Duchamp, G. and Krob, D. (1994). Combinatorics in trace monoids II. In: Diekert and Rozenberg, editors, The Book of Traces.

[8] Nijenhuis, A. and Wilf, H. S. (1975). Combinatorial Algorithms. Academic Press.

[9] Berstel, J. and Pocchiola, M. (1992). Average cost of Duval's algorithm for generating Lyndon words. Preprint no. 92-8, Laboratoire d'Informatique de l'Ecole Normale Supérieure, Paris. 